\title{
LA IMPORTANCIA DE DESARROLLAR LA INTELIGENCIA EMOCIONAL EN EL PROFESORADO
}

\author{
Natalio Extremera y Pablo Fernández-Berrocal \\ Universidad de Málaga, España
}

\section{INTRODUCCIÓN}

Educar la inteligencia emocional de los estudiantes se ha convertido en una tarea necesaria en el ámbito educativo y la mayoría de los docentes considera primordial el dominio de estas habilidades para el desarrollo evolutivo y socio-emocional de sus alumnos. En otro lugar, se ha defendido y desarrollado la importancia de desarrollar en el alumnado las habilidades relacionadas con la inteligencia emocional en el ámbito educativo (Fernández-Berrocal y Extremera, 2002), se ha realizado una revisión de las medidas actuales de evaluación de la inteligencia emocional que son aplicables al aula (Extremera y FernándezBerrocal, 2003a) y se ha llevado a cabo una recopilación de los principales hallazgos científicos que vinculan una adecuada inteligencia emocional con mejores niveles de ajuste psicológico (Extremera y Fernández-Berrocal, 2003b; Extremera y Fernández-Berrocal, en revisión). Sin embargo, a veces se piensa de forma errónea que las competencias afectivas y emocionales no son imprescindibles en el profesorado. Mientras que para enseñar matemática o geografía el profesor debe poseer conocimientos y actitudes hacia la enseñanza de esas materias, las habilidades emocionales, afectivas y sociales que el profesor debe incentivar en el alumnado también deberían ser enseñadas por un equipo docente que domine dichas capacidades. En este artículo analizaremos algunos datos que demuestran que la inteligencia emocional es un conjunto de habilidades que el docente debería aprender por dos razones: (1) porque las aulas son el modelo de aprendizaje socio-emocional adulto de mayor impacto para los alumnos y (2) porque la investigación está demostrando que unos adecuados niveles de inteligencia emocional ayudan a afrontar con mayor éxito las contratiempos cotidianos y el estrés laboral al que se enfrentan los profesores en el contexto educativo.

\section{EL DOCENTE COMO MODELO Y PROMOTOR DE LA INTELIGENCIA EMOCIONAL DEL ALUMNO}

Una de las razones por la que el docente debería poseer ciertas habilidades emocionales tiene un marcado cariz altruista y una finalidad claramente educativa. Para que el alumno aprenda y desarrolle las habilidades emocionales y afectivas relacionadas con el uso inteligente de sus emociones necesita de un "educador emocional". El alumno pasa en las aulas gran parte de su infancia y adolescencia, periodos en los que se produce principalmente el desarrollo emocional del niño, de forma que el entorno escolar se configura como un espacio privilegiado de socialización emocional y el profesor/tutor se convierte en su referente más importante en cuanto actitudes, comportamientos, emociones y sentimientos. El docente, lo quiera o no, es un agente activo de desarrollo afectivo y debería hacer un uso consciente de estas habilidades en su trabajo.

Los profesores son un modelo adulto a seguir por sus alumnos en tanto son la figura que posee el conocimiento, pero también la forma ideal de ver, razonar y reaccionar ante la vida. El profesor, sobretodo 
en los ciclos de enseñanza primaria, llegará a asumir para el alumno el rol de padre/madre y será un modelo de inteligencia emocional insustituible. Junto con la enseñanza de conocimientos teóricos y valores cívicos al profesor le corresponde otra faceta igual de importante: moldear y ajustar en clase el perfil afectivo y emocional de sus alumnos. De forma casi invisible, la práctica docente de cualquier profesor implica actividades como (Abarca, Marzo y Sala, 2002; Vallés y Vallés, 2003):

- la estimulación afectiva y la expresión regulada de los sentimientos positivos y, más difícil aún, de las emociones negativas (e.g., ira, envidia, celos,...);

- la creación de ambientes (tareas escolares, dinámicas de trabajo en grupo,...) que desarrollen las capacidades socio-emocionales y la solución de conflictos interpersonales;

- la exposición a experiencias que puedan resolverse mediante estrategias emocionales;

- o la enseñanza de habilidades empáticas mostrando a los alumnos cómo prestar atención y saber escuchar y comprender los puntos de vista de los demás.

Por otro lado, tampoco podemos dejar toda la responsabilidad del desarrollo socio-afectivo del alumno en manos de los docentes, especialmente cuando la familia es un modelo emocional básico y conforma el primer espacio de socialización y educación emocional del niño. Además, incluso cuando el profesorado se encuentra concienciado de la necesidad de trabajar la educación emocional en el aula, en la mayoría de las ocasiones los profesores no disponen de la formación adecuada, ni de los medios suficientes para desarrollar esta labor y sus esfuerzos con frecuencia se centran en el diálogo moralizante ante el cual el alumno responde con una actitud pasiva (Abarca et al., 2002). Por esta razón, padres y profesores deben complementarse en estas tareas $y$, de forma conjunta, proporcionar oportunidades para mejorar el perfil emocional del alumno. Así pues, los padres en la relación con sus hijos deben adoptar lo que se conoce como un estilo educativo democrático en contraposición a otros menos beneficiosos como son el estilo autoritario, permisivo o de no-implicación. El estilo democrático requiere por parte de los padres una exigencia pero también una receptividad. Por un lado deben exigir el cumplimiento de las demandas acordes a la madurez del niño, pero a la vez incentivar la toma de decisiones, mostrar cariño y escuchar las opiniones del niño y conocer sus gustos y preferencias. Esto implica participar de forma activa en el tiempo que pasan en casa con sus hijos, conocer el tipo de juegos que practican, los programas televisivos o vídeos que ven, aconsejar sobre los libros que podrían leer, saber los amigos con los que juegan o salen, interesarse por la música que escuchan, o supervisar el acceso a Internet. Este tipo de actividades construye la vida emocional del niño o adolescente y en muchas ocasiones, por diversos motivos, los padres son los grandes ausentes. En el aula, por su parte, los profesores y educadores determinan tareas de similar valor afectivo y emocional. Cada vez más la sociedad y las administraciones educativas son conscientes de la necesidad de un curriculum específico que desarrolle contenidos emocionales. Sin embargo, mientras estas actividades y estrategias pedagógicas no se concreten en un curriculum reglado establecido en el sistema educativo, la única esperanza para nuestros alumnos es confiar en la suerte y que su profesor/ra sea un modelo emocional eficaz y una fuente de aprendizaje afectivo adecuado a través de su influencia directa. Las interacciones profesor-alumno son un espacio socio-emocional ideal para la educación emocional con actividades cotidianas como:

- Contar problemas o intercambiar opiniones y consejos, 
- la mediación en la resolución de conflictos interpersonales entre alumnos,

- las anécdotas que pueda contar el propio profesor sobre cómo resolvió problemas similares a los que pasan los alumnos, o

- la creación de tareas que permitan vivenciar y aprender sobre los sentimientos humanos como la proyección de películas, la lectura de poesía y narraciones, las representaciones teatrales. EI contenido artístico relacionado con la música y la pintura y su posterior debate en clase desempeñan una función emocional esencial (para una experiencia docente con la poesía y el desarrollo emocional ver Doreste, 2002 y también El baúl de las emociones, http://www.juntadeandalucia.es/averroes/ cephu3/emociones/ ).

Con estas actividades el alumno descubre la diversidad emocional, fomenta su percepción y comprensión de los sentimientos propios y ajenos, observa cómo los sentimientos motivan distintos comportamientos, percibe la transición de un estado emocional a otro (e.g., del amor al odio), es consciente de la posibilidad de sentir emociones contrapuestas (e.g., sorpresa e ira, felicidad y tristeza) y cómo los personajes literarios o de cine resuelven sus conflictos o dilemas personales (Mayer y Salovey, 1997; Mayer y Cobb, 2000; Sanz y Sanz, 1997). El objetivo final es que con la práctica lleguen a trasladar estas formas de tratar y manejar las emociones a su vida cotidiana, aprendiendo a reconocer y comprender los sentimientos de los otros alumnos o profesores, empatizando con las emociones de los demás compañeros de clase, regulando su propio estrés y/o malestar, optando por resolver y hacer frente a los problemas sin recurrir a la violencia. En definitiva, enseñando a los alumnos a prevenir comportamientos violentos, desajustados emocionalmente, tanto fuera como dentro del aula. Una serie de actividades para padres y profesores encaminadas a potenciar la percepción, comprensión y regulación emocional propia y también la de sus hijos/alumnos puede encontrarse en Fernández-Berrocal y Ramos (2004).

En esta línea es donde creemos que la inteligencia emocional aporta los cimientos para el desarrollo de otras competencias más elaboradas (Fernández-Berrocal y Ramos, 2002). De hecho, algunos autores diferencian entre las habilidades básicas de inteligencia emocional y otro tipo de competencias emocionales y sociales más generales (Cherniss, 2001). El modelo de inteligencia emocional de Mayer y Salovey propone una serie de habilidades básicas tales como la percepción, la asimilación, la comprensión y la regulación emocional (Mayer y Salovey, 1997). Una vez desarrolladas estas habilidades estamos preparados para adquirir competencias más complejas que aparecen reflejadas en muchos programas transversales de prevención de consumo de drogas, de educación sexual, formación moral y cívica, etc. Por ejemplo, desarrollar la habilidad básica para reconocer las emociones en otras personas ayuda a fomentar competencias específicas para resolver con eficacia una eminente pelea con otro compañero de clase. La capacidad de asimilar un estado emocional (e.g., la felicidad) a nuestros pensamientos puede modificar la perspectiva hacia los problemas (e.g., adoptar una visión más optimista) y cambiar los puntos de vista (e.g., reevaluación de la situación) mejorando o ampliando las formas de solucionar los contratiempos. Por otro lado, el desarrollo de nuestra habilidad de comprensión emocional nos puede ayudar, por ejemplo, a entender las dificultades y sacrificios que en muchos casos supone conseguir una meta (e.g., aprobar el curso académico) y captar las consecuencias positivas que puede tener a medio plazo proseguir en la consecución de un objetivo difícil (e.g., conseguir algo prometido en verano; poder optar al modulo profesional deseado). Finalmente, aquellos alumnos que sean capaces de regular sus emociones negativas y mantener las positivas les será más fácil desarrollar competencias más elaboradas relacionadas con la 
tolerancia a la frustración o la asertividad (e.g., aceptar las críticas, defender tu postura de forma no agresiva,...).

Algunos programas educativos anglosajones (también en España, Vallés, 2003) enfatizan las habilidades integrantes del concepto de inteligencia emocional propuesto por Mayer y Salovey como una herramienta útil para el afrontamiento de la conflictividad personal e interpersonal de los centros de Educación Secundaria así como para el desarrollo integral del alumno. No obstante, aún estamos empezando a descubrir la relevancia e influencia del mundo emocional en el aula y, a pesar de que el conocimiento afectivo está muy relacionado con la madurez general, la autonomía y la competencia social del niño, son muy pocas las instituciones educativas que tienen establecidos programas específicos con estos contenidos y que fomenten en el profesorado las habilidades necesarias para llevar a cabo un estilo educativo que enfatice el desarrollo emocional. Por ejemplo, Sala (2002) ha constatado en una muestra de futuros educadores que las competencias emocionales, evaluadas con una medida de auto-informe de inteligencia emocional (Emotional Quotient Inventory, EQ-i), inciden de forma moderada en los estilos educativos, creencias, actitudes y valores de estos futuros profesionales de la docencia. Así, un estilo educativo sobreprotector se relacionaba con menores habilidades intrapersonales de inteligencia emocional, el estilo punitivo se relacionaba con niveles más bajos de habilidades interpersonales de inteligencia emocional y el perfil asertivo se relacionó con un buen estado afectivo general.

En definitiva, como apuntan Valles y Valles (2003) la educación emocional debería estar inserta en las distintas áreas curriculares, no en cuanto a su enseñanza/aprendizaje como contenido de cada área, sino cómo estilo educativo del docente que debe transmitir modelos emocionales adecuados en los momentos en los que profesor y alumno conviven en el aula.

\section{LA INTELIGENCIA EMOCIONAL COMO FACTOR PROTECTOR DEL ESTRÉS DOCENTE}

Existe una segunda razón más interesada y egoísta por la que las habilidades de inteligencia emocional son importantes en el profesorado. En concreto, las habilidades de inteligencia emocional ejercen efectos beneficiosos para el profesorado a nivel preventivo. Es decir, la capacidad para razonar sobre nuestras emociones, percibirlas y comprenderlas, como habilidad intrínseca del ser humano, implica, en último término, el desarrollo de procesos de regulación emocional que ayudarían a moderar y prevenir los efectos negativos del estrés docente a los que los profesores están expuesto diariamente.

Pero, ¿por qué es necesario aprender a razonar, comprender y regular nuestras emociones en el contexto educativo? El motivo es que la actividad docente es una de las profesiones con mayor riesgo de padecer distintas enfermedades. Resumidamente, es una cruda realidad que los docentes hoy en día experimentan de forma cada vez más creciente una variedad de trastornos y síntomas relacionados con la ansiedad, la ira, la depresión y el conocido síndrome de estar quemado o burnout. Estos problemas de salud mental además se agravan, en algunos casos, con la aparición de diferentes alteraciones fisiológicas (e.g., úlceras, insomnio, dolores de cabeza tensionales,...) como consecuencia de diversos estresores en el ámbito laboral que van articulando su aparición y desarrollo (Durán, Extremera y Rey, 2001). En la actualidad, los profesores han de afrontar una posición diferente, nuevos retos y desafíos que poco tienen que ver con los de décadas anteriores. Aspectos como la falta de disciplina del alumnado, problemas de comportamiento, el excesivo número de alumnos, la falta de motivación por aprender, la apatía estudiantil 
por realizar las tareas escolares encomendadas y el bajo rendimiento se han convertido en importantes fuentes de estrés para el profesorado que afectan a su rendimiento laboral. La inmigración está contribuyendo a la heterogeneidad cultural de las clases, lo cual se convierte en un desafío adicional para el profesor que debe ajustar el estilo de enseñanza y el curriculum a las nuevas necesidades convirtiéndose en un nuevo factor de estrés (Tatar y Horenczyk, 2003). En muchos casos, la pérdida de credibilidad en la labor profesional de los profesores y el bajo estatus social y profesional, entre otros, merman aún más la capacidad de afrontamiento del docente. Así, la sociedad demanda al profesor una mayor preparación técnica, especialización no sólo en contenidos sino también en la metodología docente, en el conocimiento psicológico de los alumnos, la enseñanza de valores cívicos y morales, etc. A estas crecientes exigencias se suma el hecho de que el sistema educativo no siempre favorece un contexto organizacional que apoye al profesor, independientemente del nivel en el que se sitúe. Como se observa, mientras los profesores se incorporan a su profesiones con unas altas expectativas y con un objetivo claro de educar a los niños y adolescentes. Las diversas fuentes de estrés existentes van degradando esa expectativa original. Las condiciones laborales, la falta de recursos en comparación con las altas demandas requeridas, las distintas presiones temporales se convierten en obstáculos, muchas veces insalvables, que pueden hundir el entusiasmo inicial del docente y desembocar en la aparición de estrés laboral, diversos síntomas ansiosos o depresivos y trastornos de salud física y mental dando lugar, en algunos casos, al absentismo, la baja laboral o el abandono de la institución.

Las consecuencias de esta situación, finalmente, no afectarán únicamente al profesional docente (e.g., bajo bienestar psicológico, deterioro de las relaciones sociales), o a la organización en la que trabaja (e.g., absentismo, abandono de la institución), sino que el alumno/a va a ser el directo receptor de un servicio de "baja calidad" en relación a algo esencial: su propia educación y/o formación como profesional.

Debido a la ingente cantidad de factores que ocurren durante la práctica docente, resulta difícil atribuir a una o varias causas el síndrome del burnout docente y sus consecuencias. No obstante, se reconocen varios grupos de agentes que contribuyen a la aparición del estrés laboral en el profesorado. Entre estos factores podemos destacar tres grandes grupos: 1) factores que se sitúan en el contexto organizacional y social (e.g., sobrecarga de trabajo; presiones temporales, escasez de recursos,...; 2) factores vinculados a la relación educativa (e.g., escasa disciplina y mala conducta al alumnado, desmotivación estudiantil, falta de comprensión por parte de compañeros de trabajo); y 3) factores personales e individuales relacionado con variables inherentes del profesorado que influyen en la vulnerabilidad al estrés docente (e.g., experiencia docente, autoestima, estilo atribucional, características de personalidad) (Doménech, 1995; Valero, 1997). Las habilidades de inteligencia emocional se centran en este tercer grupo de factores relacionados con las habilidades intrínsecas del docente. La literatura ha dejando patente la relación entre una mayor vulnerabilidad hacia el síndrome de burnout y diversas características de personalidad tales como autoestima, locus de control, patrón tipo A, etc (Schaufeli y Enzmann, 1998; Maslach, Schaufeli, \& Leiter, 2001). El problema de estas variables clásicas de personalidad es que hacen referencia a rasgos o estados inherentes de la persona más que a la manera en la cual el sujeto percibe, comprende y maneja sus emociones y la de los demás. En este sentido, desde el campo de estudio de la inteligencia emocional se empieza a prestar atención a las habilidades emocionales que las personas desarrollan para afrontar los diversos contratiempos, entre ellos, aquellos ocurridos en el contexto escolar. Puesto que lo característico y peculiar del burnout es que el componente estresante surge 
de la interacción social entre quien ofrece sus servicios y quien los recibe, examinar diferencias individuales en las habilidades para regular las emociones negativas de los demás, así como manejar las propias sería un componente clave en el estudio de los recursos personales que hacen al profesorado más resistente a la aparición del síndrome.

Algunos trabajos recientes han encontrado evidencias de que una gestión adecuada de nuestras reacciones emocionales disminuye los niveles globales de estrés laboral del profesorado incluso cuando se controlan estresores típicamente organizacionales y del entorno de trabajo. Por ejemplo, Mearns y Cain (2003) encontraron que profesores estadounidenses, tanto de secundaria como de primaria, con altas expectativas sobre la regulación de sus emociones negativas utilizaban más estrategias de afrontamiento activo para enfrentarse a las situaciones laborales estresantes, experimentaban menos consecuencias negativas del estrés y sus puntuaciones en su realización personal por el trabajo eran mayores.

Otros estudios en España han examinado el papel de la inteligencia emocional, evaluada a través de una medida de auto-informe (Trait Meta-Mood Scale (TMMS)), y ciertas estrategias de afrontamiento como la supresión de pensamientos en la aparición del burnout y el desajuste emocional en profesores de enseñanza secundaria (Extremera, Fernández-Berrocal y Durán, 2003). Los resultados revelaron que los niveles de inteligencia emocional auto-informados de los docentes y la tendencia a suprimir los pensamientos negativos explicaban parte de la varianza de las dimensiones de burnout no explicada por la edad, el sexo o los años de docencia de los profesores, factores, por otra parte, clásicamente relacionados con el síndrome. En concreto, para la variable de salud mental y cada una de las dimensiones del burnout los resultados fueron los siguientes: el profesorado con una mayor tendencia a suprimir sus pensamientos negativos y menor capacidad de reparación emocional indicaban un mayor cansancio emocional, mayores niveles de despersonalización y puntuaciones más bajas en salud mental. En cambio, aquellos docentes con una capacidad más elevada para reparar y discriminar sus estados emocionales informaron puntuaciones más elevadas en su realización personal.

Finalmente, se dividieron a los profesores en grupos extremos (altas y bajas puntuaciones en cada dimensión de burnout). El grupo con alto cansancio emocional se diferenció del grupo con bajo cansancio en puntuaciones más elevadas en atención emocional y supresión de pensamientos y niveles más bajos de reparación y salud mental. Con respecto al grupo de profesores caracterizado por alta despersonalización, presentaban menores niveles de reparación y mayor tendencia a suprimir sus pensamientos. Finalmente, el grupo de docentes con altos niveles de realización personal presentaba puntuaciones más elevadas en claridad emocional y reparación de las emociones.

Utilizando una medida de habilidad de inteligencia emocional hemos encontrado resultados similares. En concreto, en un estudio con 42 profesores de secundaria de dos institutos diferentes observamos relaciones significativas entre la escala de manejo emocional del MSCEIT (Mayer Salovey Caruso Emotional Intelligence Test; Mayer, Salovey y Caruso, 2002) y puntuaciones en burnout y salud mental. Las puntuaciones en la escala de manejo emocional se relacionaban positivamente con salud mental y con la realización personal del profesorado, mientras que correlacionaba de forma negativa con despersonalización. Por otra parte, la escala de manejo emocional del MSCEIT explicaba varianza significativa en salud mental y en las subescalas de burnout, incluso cuando se controlaron variables sociodemográficas como el sexo, la edad y los niveles percibidos de reparación emocional del TMMS-24 (Extremera, Fernández-Berrocal, Lopes, y Salovey, en preparación). 
Estos hallazgos muestran que la inteligencia emocional proporciona un marco teórico prometedor para conocer los procesos emocionales básicos que subyacen al desarrollo del burnout y puede ayudar a comprender mejor el rol mediador de ciertas variables emocionales del profesorado y su influencia en el rendimiento y la calidad de sus servicios en el aula y de su propio bienestar personal. Asimismo, estos resultados subrayan la importancia de las habilidades emocionales de las personas para afrontar inteligentemente situaciones altamente afectivas que ponen al docente al límite de sus recursos. Tales habilidades deben ser tenidas en cuenta para prevenir la aparición del síndrome del burnout en la práctica docente y, sobretodo, en futuros programas dirigidos a la prevención, formación y entrenamiento en el control del estrés laboral del profesorado.

Para finalizar, no queremos hacer creer al lector que la inteligencia emocional cambiará drásticamente su vida a partir de hoy. Debemos ser realistas, estas habilidades no nos convertirán en invulnerables ni nos prevendrán del desconcierto. Nuestra inteligencia emocional no hará que nuestros alumnos sean educados y estén motivados por aprender, que nuestros compañeros o padres de alumnos no discutan con nosotros o que nuestros problemas burocráticos y administrativos del centro se acaben. Ahora bien, ser emocionalmente inteligente disminuirá el desgaste psicológico que implica todo este tipo de problemas diarios y facilitará nuestra tarea en el aula e, incluso, hará que volvamos a disfrutar de una tarea tan fascinante como es enseñar a los demás.

\section{CONCLUSIONES}

En este artículo hemos revisado la necesidad y las implicaciones de desarrollar las habilidades de inteligencia emocional del profesorado. Hasta la fecha, la mayoría de trabajos aparecidos estaban centrados en el fomento de la inteligencia emocional y en sus repercusiones sobre la vida escolar y personal de los alumnos.

Desde nuestro punto de vista, la importancia de estas habilidades también se traslada a la otra parte que constituye el proceso de enseñanza/aprendizaje: el profesorado. El conocimiento emocional del docente es un aspecto fundamental para el aprendizaje y el desarrollo de estas competencias en los alumnos porque el profesor se convierte en un modelo de aprendizaje vicario a través del cual el alumno aprende a razonar, expresar, y regular todas esas pequeñas incidencias y frustraciones que transcurren durante el largo proceso de aprendizaje en el aula. El desarrollo de las habilidades de inteligencia emocional en el profesorado no sólo servirá para conseguir alumnos emocionalmente más preparados, sino que además ayudará al propio profesor a adquirir habilidades de afrontamiento. De este modo, los docentes emocionalmente más inteligentes, es decir, aquellos con una mayor capacidad para percibir, comprender y regular las emociones propias y la de los demás, tendrán los recursos necesarios para afrontar mejor los eventos estresantes de tipo laboral y manejar más adecuadamente las respuestas emocionales negativas que frecuentemente surgen en las interacciones que mantienen con los compañeros de trabajo, los padres y los propios alumnos. A partir de ahora, los docentes tendrán una razón más para aprender las matemáticas de los sentimientos y el lenguaje de las emociones.

\section{REFERENCIAS}

AbARCA, M., MARzo, L., y SALA, L. (2002). La educación emocional en la práctica educativa de primaria. Bordón, 54, 505-518. 
CHERNISS, C. (2001). Emotional intelligence and the good community. American Journal of Community Psychology, 30, 1-11.

DOMÉNECH, D.B. (1995). Introducción al síndrome "burnout" en profesores y maestros y su abordaje terapéutico. Psicología Educativa, 1, 63-78.

DORESTE, J (2002). La educación de las emociones a través de la lectura. En P. Fernández-Berrocal y N. Ramos Díaz (Eds). Corazones Inteligentes (pp. 377-393). Barcelona: Kairós.

DurÁn, A., y Extremera, N. Rey, L. (2001). Burnout en profesionales de la enseñanza: un estudio en Educación primaria, secundaria y superior. Revista de Psicología del Trabajo y de las Organizaciones, 17, 45-62.

ExtRemera N. y FernándeZ-BerRoCAL, P (2002). Educando emociones: La educación de la inteligencia emocional en la escuela y la familia. En P. Fernández-Berrocal y N. Ramos Díaz (Eds). Corazones Inteligentes (pp. 353-375). Barcelona: Kairos.

EXTREMERA, N. y FERnÁNDEZ-BERROCAL, P. (2003a). La inteligencia emocional: Métodos de evaluación en el aula. Revista Iberoamericana de Educación, 30, 1-12.

EXtremera, N., y Fernández-BerRocal, P. (2003b). La inteligencia emocional en el contexto educativo: hallazgos científicos de sus efectos en el aula. Revista de Educación, 332, 97-116.

Extremera, N. y Fernández-Berrocal, P. (en revisión). El papel de la inteligencia emocional en el alumnado: evidencias empíricas. Revista Electrónica de Investigación Educativa.

Extremera, N. y Fernández-BerRocal, P. y Durán, A. (2003). Inteligencia emocional y burnout en profesores. Encuentros en Psicología Social, 1, 260-265.

Extremera, N., Fernández-Berrocal, P., Lopes, P. N., y Salovey, P. (en preparación). Managing emotions, burnout and mental health in secondary school teachers.

FERNÁNDEZ-BERRoCAL, P. y EXTREMERA, N. (2002). La inteligencia emocional como una habilidad esencial en la escuela. Revista Iberoamericana de Educación, 29, 1-6.

FERnÁNDEZ-BerRocal, P. y RAmOs, N. (2002). Corazones Inteligentes. Barcelona: Kairós.

FERNÁNDEZ-BERROCAL, P. y RAMOS, N. (2004). Desarrolla tu inteligencia emocional. Barcelona: Kairós.

MASLACH, C., SchaUfeli, W.B. y LeiteR, M.P. (2001). Job burnout. Annual Review of Psychology, 52, 397422.

MAYER, J. D. y SAlovey, P. (1997). What is emotional intelligence? En P. Salovey \& D. Sluyter (Eds). Emotional Development and Emotional Intelligence: Implications for Educators (pp. 3-31). New York: Basic Books.

Mayer, J. D., Salovey, P., y Caruso, D. (2002). Mayer-Salovey-Caruso Emotional Intelligence Test (MSCEIT): User's Manual. Toronto: Multi-Health Systems, Inc.

MAYER, J. D., y СовB, C. D. (2000). Educational policy on emotional intelligence: Does it make sense? Educational Psychology Review, 12, 163-183. 
MEARNS, J., y CAIN, J. E. (2003). Relationships between teachers' occupational stress and their burnout and distress: roles of coping and negative mood regulation expectancies. Anxiety, Stress and Coping, 16, 71-82.

SALA, J. (2002). Ideas previas sobre la docencia y competencias emocionales en estudiantes de Ciencias de la Educación. Revista Española de Pedagogía, 223, 543-557.

SANZ de Acedo, M. L. L. y SANZ de Acedo, R. L. (1997). La educación infantil y la inteligencia emocional. Comunidad educativa, 2, 44-47.

Schaufeli, W. B. y Enzmann, D. (1998). The Burnout Companion to Study and Practice: A Critical Analysis. London: Taylor \& Francis.

TATAR, M., y HORENCZYK, G. (2003). Diversity-related burnout among teachers. Teaching and Teacher Education, 19, 397-408.

VALERO, L. (1997). Comportamientos bajo presión: El burnout en los educadores. En Ma..l. Hombrados (Ed.). Estrés y Salud (213-237). Promolibro: Valencia.

VALLÉs, A. (2003). Emoción-ate con inteligencia. Valencia: Promolibro.

VAlLÉS, A. y VALLÉS, C. (2003). Psicopedagogía de la Inteligencia Emocional. Valencia: Promolibro. 